\title{
Bile Duct Involvement by Hepatocellular Carcinoma: A Rare Occurrence and Poor Prognostic Indicator in Bile Duct Brushing Samples
}

\author{
Shristi Bhattarai'; Rondell P. Graham, MBBS²; Carlie S. Sigel, MD iD 3; Jiaqi Shi, MD ${ }^{4}$; \\ Raul S. Gonzalez, MD ${ }^{5}$; Yue Xue, MD ${ }^{6}$; Alyssa M. Krasinskas, MD ${ }^{6}$; Kim HooKim, MD; Volkan Adsay, MD ${ }^{8}$; \\ and Michelle D. Reid, MD iD 6
}

\begin{abstract}
BACKGROUND: Hepatocellular carcinoma (HCC) rarely involves the biliary tree and may be inadvertently sampled on bile duct brushings (BDBs). METHODS: The pathology archives of 5 institutions were searched for BDBs with HCC involvement. RESULTS: A total of 17 BDBs from 14 patients were obtained. There was a male:female ratio of 6:1; the median age of the patients was 59.5 years (range, 22-80 years). The median hepatic tumor size was $6.2 \mathrm{~cm}$ (range, $2.2-13.0 \mathrm{~cm}$ ). $\mathrm{HCC}$ risk factors included viral hepatitis ( 5 patients), cirrhosis ( 5 patients), hemochromatosis ( 1 patient), and alcoholic steatohepatitis (1 patient). Jaundice with elevated bilirubin, liver enzymes, and a-fetoprotein was common. Endoscopic retrograde cholangiopancreatography demonstrated bile duct dilatation, polypoid intraductal masses (5 samples), clots/debris (2 samples), or strictures (4 samples). All BDBs had single and clustered large cells with naked atypical nuclei, granular cytoplasm, high nuclear/cytoplasmic ratios, and nuclei with prominent macronucleoli. Less common findings included clear/microvesicular cytoplasm (35\%), papillae (29\%), and anisonucleosis (35\%). Classic HCC features (widened trabeculae [35\%], endothelial wrapping [24\%], multinucleation [24\%], and cytoplasmic bile pigment [35\%]) were uncommon. A total of 11 BDBs were diagnosed as malignant (10 with $\mathrm{HCC}$ and 1 with cholangiocarcinoma), 2 were diagnosed as atypical, and 1 BDB was diagnosed as negative; approximately two-thirds were found to have polysomy on fluorescence in situ hybridization. Approximately $71 \%$ of patients died of disease at a median of 3.5 months. CONCLUSIONS: HCC may extend into the intrahepatic and/or extrahepatic biliary tree, causing masses and/or strictures that may be sampled on BDB. Although cytologically malignant, the classic features of HCC are uncommon, which can cause misdiagnosis. Cytopathologists should be mindful of this differential when evaluating BDBs, particularly when concomitant liver masses and/or HCC risk factors are present. Because of the associated high mortality and rapid rate of death, its presence should be conveyed clearly in pathology reports. Cancer Cytopathol 2019;127:691-699. (c) 2019 American Cancer Society.
\end{abstract}

KEY WORDS: bile duct; brushing; carcinoma; hepatocellular.

\section{INTRODUCTION}

The diagnosis of neoplasms in the biliary tree remains a clinical and pathologic challenge, with brush cytology and (less frequently) small biopsies being the most frequently used, albeit imperfect, diagnostic tests. The cytologic diagnosis of malignancy often is confounded by well-differentiated cytologically bland carcinoma, as well as instrumentation-related and cholangitis-related reactive changes. ${ }^{1,2}$

\footnotetext{
Corresponding author: Michelle D. Reid, MD, Department of Pathology, Rm G179, Emory University Hospital, 1364 Clifton Rd NE, Atlanta, GA 30322; michelle.reid@emory.edu

${ }^{1}$ Department of Biology, Georgia State University, Atlanta, Georgia; ${ }^{2}$ Department of Pathology, Mayo Clinic, Rochester, Minnesota; ${ }^{3}$ Department of Pathology, Memorial Sloan Kettering Cancer Center, New York, New York; ${ }^{4}$ Department of Pathology, University of Michigan, Ann Arbor, Michigan; ${ }^{5}$ Department of Pathology, Beth Israel Deaconess Medical Center, Boston, Massachusetts; ${ }^{6}$ Department of Pathology, Emory University Hospital, Atlanta, Georgia; ${ }^{7}$ Department of Pathology, Thomas Jefferson University Hospital, Philadelphia, Pennsylvania; ${ }^{8}$ Department of Pathology, Koç University, Istanbul, Turkey
}

Received: July 26, 2019; Revised: August 26, 2019; Accepted: August 26, 2019

Published online September 13, 2019 in Wiley Online Library (wileyonlinelibrary.com)

DOI: $10.1002 /$ cncy.22185, wileyonlinelibrary.com 
Nonetheless, the majority of biliary tract tumors that are diagnosed on bile duct brushing samples (BDBs) or intraductal biopsy either are pancreatic (pancreatic ductal adenocarcinoma) or biliary (intrahepatic and extrahepatic cholangiocarcinoma) in origin. ${ }^{3-6}$ Other sources of positive results are intraductal papillary neoplasms of the bile duct, primary tumoral intraductal neoplasms of the intrahepatic and extrahepatic biliary tree, and metastatic nonpancreatobiliary tumors from the upper and lower gastrointestinal tract, and even the lung. ${ }^{4-6}$ In addition to metastases from distant sites, the biliary tract also may be involved by direct extension of hepatocellular carcinoma (HCC).

Intraductal spread of HCC into the biliary tract is an unusual source of malignant cells on BDBs. ${ }^{7-11}$ To our knowledge, the question of whether this is due to the rarity of involvement or to the failure of cytopathologists to recognize tumor in these specimens is unclear, but when contrasted with the number of new cases of HCC worldwide (estimated at 841,080 in 2018), it is surprising that this phenomenon is not encountered more frequently. ${ }^{12}$ In the current study, we sought to examine the clinicopathologic characteristics of patients with BDBs demonstrating HCC.

\section{MATERIALS AND METHODS}

A multi-institutional search of the pathology department archives of 5 large tertiary institutions (Beth Israel Deaconess Medical Center [Boston, Massachusetts], Emory University [Atlanta, Georgia; institutional review board approval IRB00095765], the Mayo Clinic [Rochester Minnesota], Memorial Sloan Kettering Cancer Center [New York City], and the University of Michigan [Ann Arbor, Michigan]) was conducted for BDBs in which HCC was identified either on initial review or on re-review triggered by a concurrent or subsequent positive liver biopsy or surgical resection. Clinicopathologic data, radiologic findings, and cytogenetic and cytohistologic features from cytology samples and concurrent or subsequent biopsies and/or surgical resections (when available) were collected for all patients. Cytology specimens were re-examined for cytologic features that historically have been described in HCC, including specimen hypercellularity, increased nuclear/cytoplasmic ratio, singly dispersed atypical naked nuclei, macronucleoli, multinucleated tumor cells, endothelial wrapping (well-defined vessels traversing tissue fragments), widened trabeculae $>2$ cell plates in thickness, and cytoplasmic bile pigment. ${ }^{13,14}$ In addition, the presence of malignant-appearing single cells with preserved granular "oncocytoid" or clear/microvesicular cytoplasm, nuclear pleomorphism or anisonucleosis, an increased nuclear/cytoplasmic ratio, papillae, necrosis, multinucleated tumor giant cells, and a 2-cell population of oncocytoid cells with abundant granular cytoplasm and ductal cells (singly dispersed columnar cells or in honeycomb sheets) was noted.

\section{RESULTS}

\section{Clinical Characteristics}

Of $>5000$ BDBs collected from the 5 institutions over $>20$ years, a total of $17 \mathrm{BDB}$ with $\mathrm{HCC}$ were identified among 14 patients. Specimen contribution by institution was 2 specimens each from the University of Michigan and the Mayo Clinic, 3 specimens from Memorial Sloan Kettering Cancer Center, 4 specimens from Beth Israel Deaconess Medical Center, and 6 specimens from Emory University. These 17 samples were identified from 12 males and 2 females (male:female ratio of 6:1) with a median age of 59.5 years (range, 22-80 years). Eight patients presented with jaundice, 13 had elevated serum bilirubin, all patients had elevated liver enzymes, 5 patients had elevated serum $\alpha$-fetoprotein levels, and 2 patients had elevated serum CA 19-9 and carcinoembryonic antigen. Risk factors for HCC were noted in 10 patients, including hepatitis $\mathrm{B}$ virus infection (2 patients), hepatitis $C$ virus infection (3 patients), hereditary hemochromatosis (1 patient), alcoholic steatohepatitis with cirrhosis (1 patient), nonalcoholic steatohepatitis (1 patient), and cirrhosis without obvious etiology (2 patients). Three patients were clinically suspected of having recurrent $\mathrm{HCC}$ at the time of BDB because they previously had been diagnosed with and successfully treated for HCC years earlier. These individuals included one patient each with hemochromatosis and alcoholic steatohepatitis and a 22-year-old patient with a history of fibrolamellar HCC.

\section{Radiologic Findings}

Imaging results (including computed tomography and magnetic resonance imaging) were available in all cases and are summarized in Table 1. A hepatic mass (or masses) was noted on imaging in 12 patients. Hepatic tumors ranged in size from $2.2 \mathrm{~cm}$ to $13.0 \mathrm{~cm}$ with a 


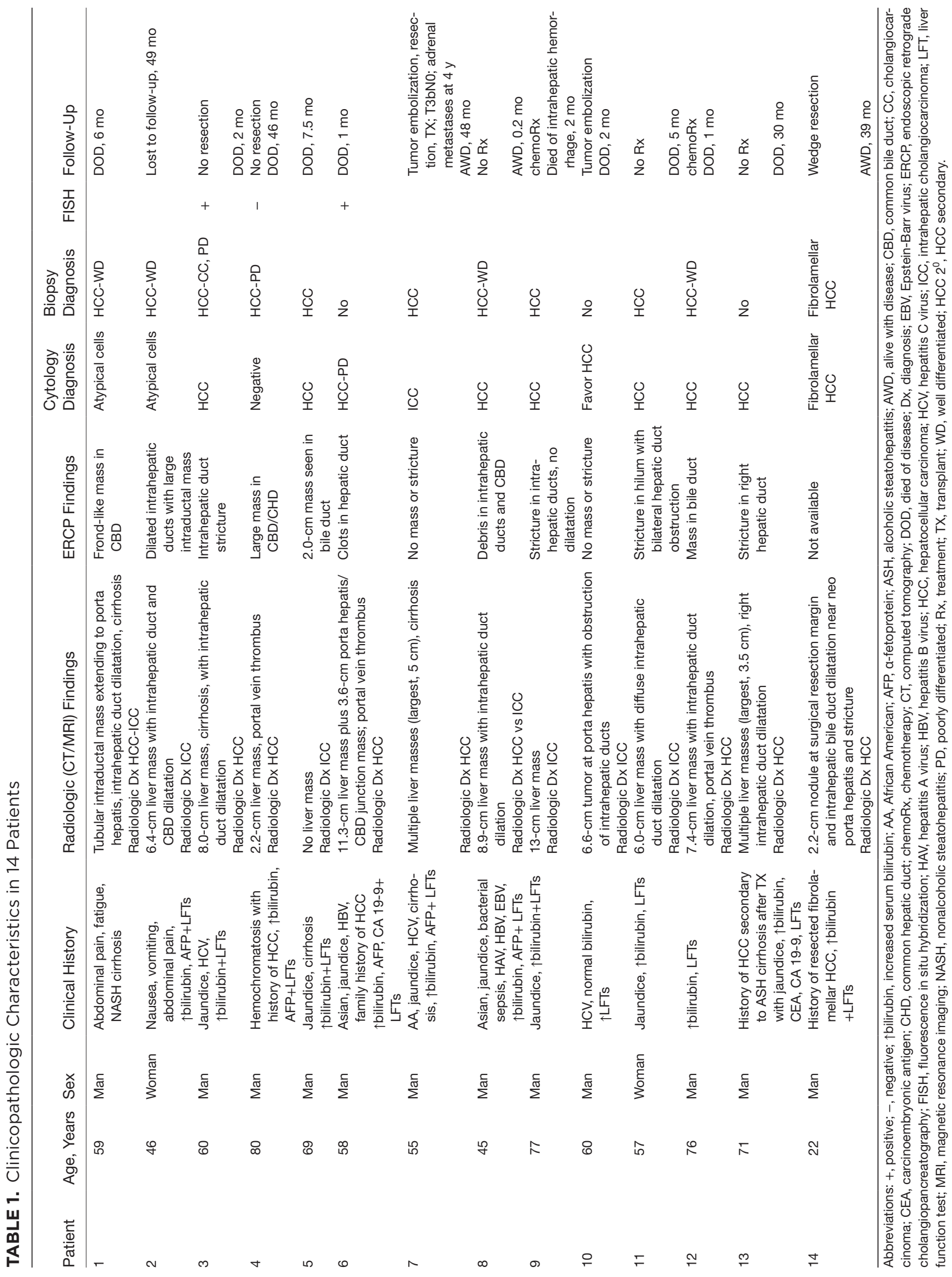




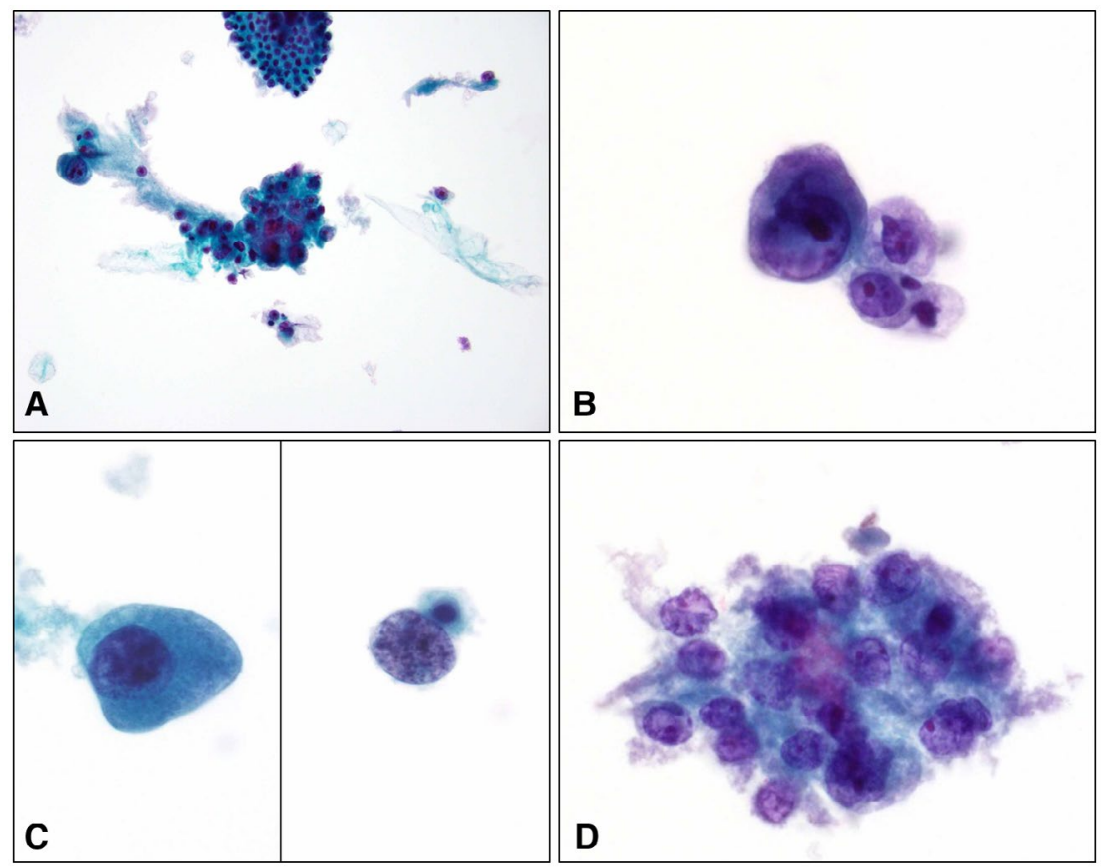

Figure 1. ThinPrep slides. (A) A mixed (2-cell) population of benign ductal cells in honeycomb sheets (Top Center) and single and clustered malignant cells (Center) with abundant granular cytoplasm, round nuclei, and cherry red macronucleoli (Papanicolaou stain, original magnification $\times 200$ ). (B) Tumor cells demonstrating a high nuclear/cytoplasmic ratio, granular to clear cytoplasm, and round hypochromatic and hyperchromatic nuclei with cherry red macronucleoli (Papanicolaou stain, original magnification $\times 600$ ). (C) Large hyperchromatic tumor cells with (Left) dense polygonal cytoplasm and (Right) naked nuclei (Papanicolaou stain, original magnification $\times 600$ ). (D) Three-dimensional cluster with hypochromasia and markedly irregular nuclear membranes. This case was misdiagnosed as adenocarcinoma (Papanicolaou stain, original magnification $\times 400$ ).

median size of $6.2 \mathrm{~cm}$. Intrahepatic and/or extrahepatic bile duct dilatation with or without involvement of the common bile duct was observed in 8 patients $(57 \%)$ and portal vein thrombosis was noted in 3 patients $(21 \%)$. Documented radiologic diagnoses included HCC (6 patients), cholangiocarcinoma (6 patients), and HCC versus intrahepatic cholangiocarcinoma (2 patients). One patient (case 5) was found to have no hepatic or bile duct masses on imaging.

\section{Endoscopic Retrograde Cholangiopancreatography Findings}

The results of endoscopic retrograde cholangiopancreatography (ERCP) were available for review in 13 patients. These demonstrated variable findings, including papillary or polypoid intraductal lesions (7 patients; described as papillary, polypoid, frond-like masses [5 patients]; debris [1 patient]; or clots [1 patient]), as well as strictures of the hepatic or common bile duct without masses ( 4 patients). Two patients were found to have no intraductal masses, strictures, or other duct abnormalities on ERCP.

\section{Cytologic Findings}

All patients had BDBs of the intrahepatic, extrahepatic, or common bile duct, and 2 patients had brushings of both the intrahepatic and common bile ducts. Sixteen samples had ThinPrep slides (14 with cell blocks and 2 without) and 1 sample had hematoxylin and eosinstained smears only. Various immunohistochemical stains (including pancytokeratin, cytokeratin 7, arginase, hepatocyte paraffin [Hep-Par], and glypican 3) and reticulin were performed on 10 specimens. Eleven specimens were termed "malignant" on cytology, one was misinterpreted as benign, and 2 specimens were classified as "atypical cells present." Of the 11 cases classified as malignant on the BDB, $9(82 \%)$ were diagnosed as HCC, 1 (9\%) as "favor HCC," and 1 (9\%) as adenocarcinoma (intrahepatic cholangiocarcinoma).

Cytologic findings that were found to be present in $80 \%$ to $100 \%$ of samples included 3-dimensional clusters and singly dispersed atypical cells with naked nuclei or abundant granular cytoplasm (Figs. 1 and 2). Cells often had a high nuclear/cytoplasmic ratio $(77 \%)$ with round to oval nuclei (nuclear irregularity was noted in 


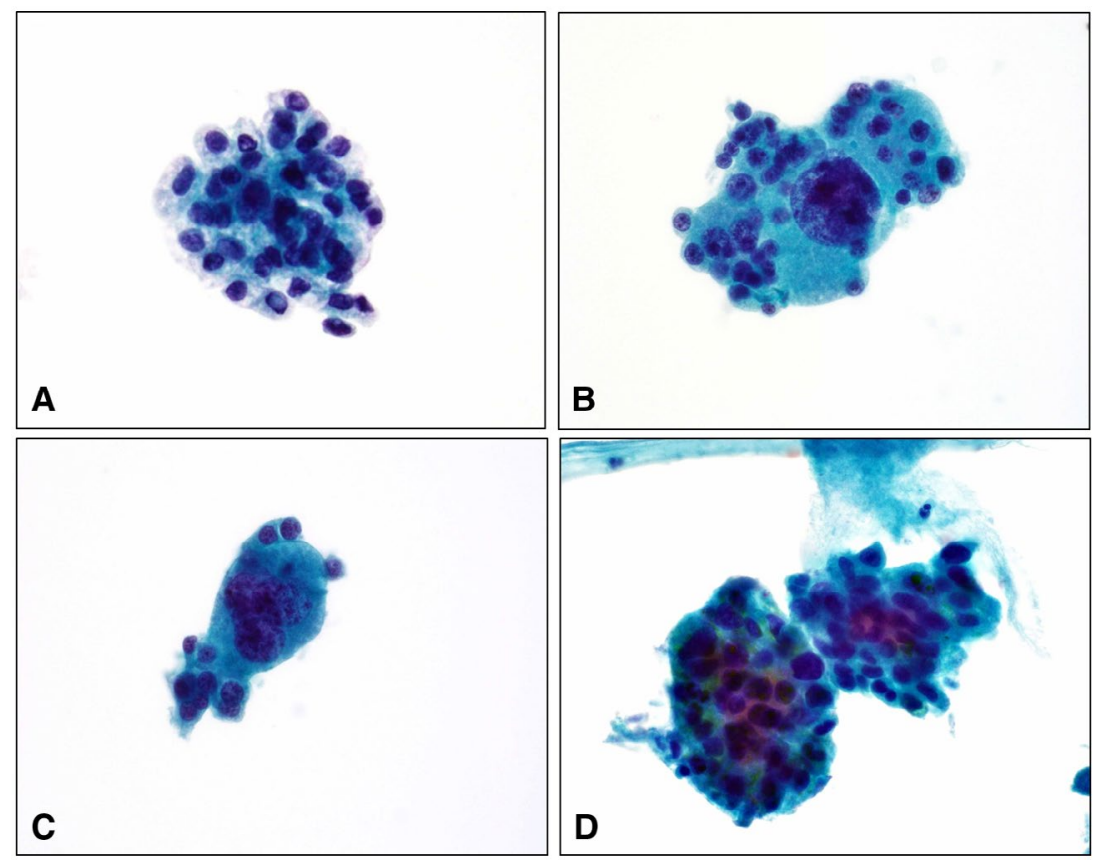

Figure 2. ThinPrep slides showing (A) a well-differentiated hepatocellular carcinoma with monotonous tumor cells with a low nuclear/cytoplasmic ratio and clear cytoplasm (Papanicolaou stain, original magnification $\times 400$ ). Examples of poorly differentiated hepatocellular carcinoma showing (B) marked ( $>5$-fold) anisonucleosis, (C) multinucleated tumor giant cells, and (D) cytoplasmic bile pigment (Papanicolaou stain, original magnification $\times 600$ ).

$35 \%$ of samples) and prominent central nucleoli were seen in the majority of cases (94\%) (Figs. 1 and 2). Less common cytologic findings included necrosis (47\%), a second population of benign-appearing ductal cells (47\%), hypercellularity (41\%), anisonucleosis of $\geq 4$-fold (35\%) (Figs. 1 and 2), clear or bubbly microvesicular pale cytoplasm (35\%), and papillary groups (29\%) (Figs. 3 and 4). Classic cytologic features of HCC such as widened trabeculae (35\%), endothelial wrapping (24\%), cytoplasmic bile pigment (24\%), and multinucleated malignant cells (24\%) were observed less frequently (Figs. 2 and 3). Widened trabeculae were best observed in cell blocks, highlighted by reticulin staining and positive for the hepatocellular differentiation markers arginase or Hep-Par (Fig. 4). Occasional cases (29\%) demonstrated variably sized "papillary groups" lined by epithelial cells with abundant eosinophilic granular to clear cytoplasm (Fig. 4), focally resembling a steatohepatitic HCC (Fig. 4). The mixed HCCcholangiocarcinoma case demonstrated single intact cells and naked atypical nuclei as well as 3-dimensional clusters of malignant cells with abundant granular or clear cytoplasm and round to oval nuclei with macronucleoli (Fig. 1) and focal nuclear membrane irregularity.
Cytologic features of the cases are highlighted in Figures $1-4$ and summarized in Table 2.

\section{Histologic Findings}

Eleven patients underwent concurrent liver biopsies at the time of BDB. The diagnosis of HCC was confirmed in 10 patients, including the fibrolamellar variant, and the tumors ranged from well to poorly differentiated. On biopsy, one tumor demonstrated a mixed HCCcholangiocarcinoma but the corresponding BDB showed only large cells with granular cytoplasm and central round nuclei with macronucleoli consistent with the HCC component. Only one tumor was resected and demonstrated a pT3b, poorly differentiated HCC with lymphovascular invasion and intraductal growth of tumor (Fig. 4).

\section{Fluorescence In Situ Hybridization}

Fluorescence in situ hybridization (FISH) was performed on 3 specimens and was "positive" in 2 cases, both of which demonstrated polysomy (one by Vysis UroVysion FISH demonstrating $>5$ cells with $\geq 2$ chromosomes [chromosomes 3, 7, and 17] and the other by a pancreatobiliary FISH probe kit that demonstrated gains of $\geq 2$ loci of $1 \mathrm{q} 21,7 \mathrm{p} 12,8 \mathrm{q} 24$, and 9p21). Both cases found to 

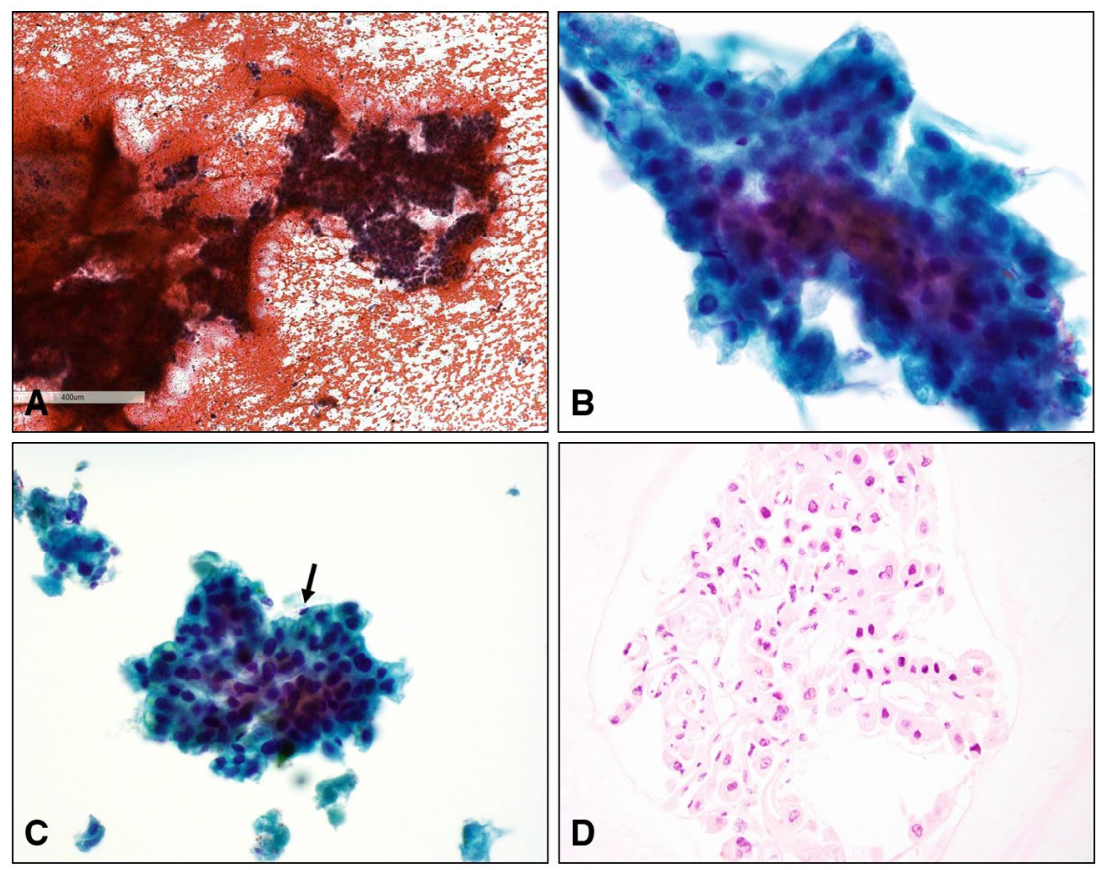

Figure 3. Hepatocellular carcinoma with tumor cells arranged in widened trabeculae $>2$ cells in thickness is shown in panels $A$ ( $H$ \& $E$, original magnification $\times 40$ ) and $B$ (Papanicolaou stain, original magnification $\times 400$ ). (C) Widened trabeculae were focally lined by flattened endothelial cells (arrow) (Papanicolaou stain, original magnification $\times 400$ ). (D) A cell block demonstrating welldifferentiated hepatocellular carcinoma (H \& E, original magnification $\times 200)$.
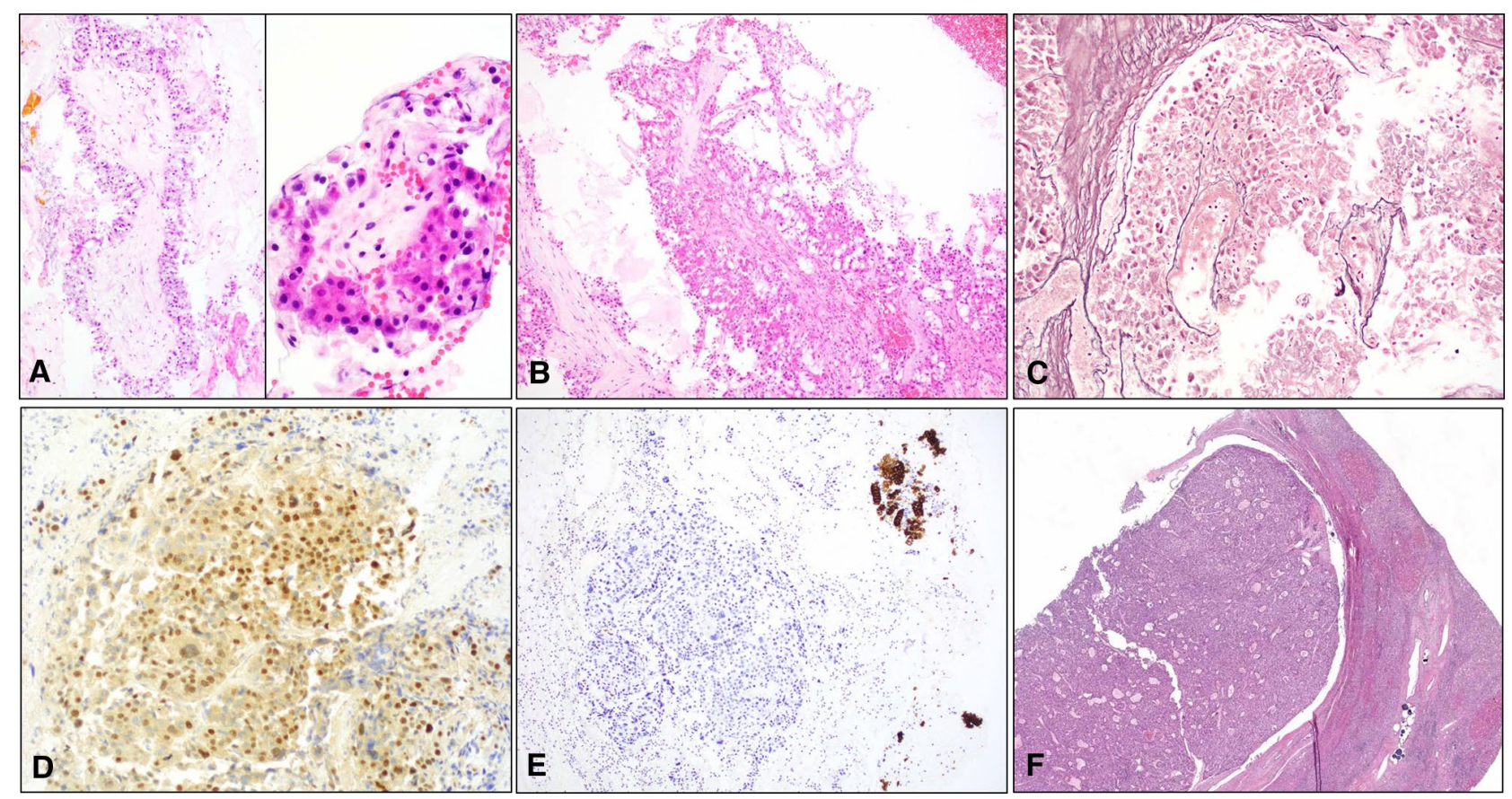

Figure 4. (A and $B$ ) Cell blocks demonstrating papillary units with central hyalinized cores lined with multilayered, large, eosinophilic to clear cells with relative monotony (H \& E, original magnification $\times 200-\times 400$ ). (C) Reticulin stain highlighting widened trabeculae (original magnification $\times 200$ ). Tumor cells were $(D)$ positive for arginase and negative for cytokeratin 7 whereas (E) the benign ductal cells (Upper Right) were positive (original magnification $\times 200$ ). (F) Hepatectomy specimen showing tumor growing as a circumscribed, nodular, intraductal mass that compressed the duct wall. Note the presence of dark blue drug-eluting bead transarterial chemoembolization (DEB TACE) spherules on the lower right ( $\mathrm{H} \& \mathrm{E}$ stain, original magnification $\times 40$ ). 
TABLE 2. Cytologic Findings in 17 Bile Duct Brushing Samples

\begin{tabular}{|c|c|c|c|c|c|c|c|c|c|c|c|c|c|c|c|c|c|c|}
\hline Feature & 1 & 2 & 3 & 4 & $5^{a}$ & $6^{a}$ & 7 & 8 & $9^{a}$ & $10^{a}$ & 11 & 12 & 13 & 14 & 15 & $16^{a}$ & $17^{a}$ & Total \\
\hline Hypercellularity & & & + & + & & & & + & + & + & & + & & & + & & & 7 (41\%) \\
\hline Single intact cells & + & + & + & + & + & + & + & + & + & + & + & + & + & + & + & + & + & $17(100 \%)$ \\
\hline Atypical naked nuclei & + & + & + & + & + & + & + & + & + & + & + & + & + & + & + & + & + & $17(100 \%)$ \\
\hline 3-dimensional clusters & + & + & + & + & + & + & + & + & + & + & + & + & + & + & + & + & + & $17(100 \%)$ \\
\hline Granular cytoplasm & + & + & + & + & + & + & + & + & + & + & + & + & + & + & + & + & + & $17(100 \%)$ \\
\hline Prominent nucleoli & + & + & + & + & + & + & + & + & & + & + & + & + & + & + & + & + & $16(94 \%)$ \\
\hline Widened trabeculae & & & & & & & & & + & + & + & & + & + & + & & & $6(35 \%)$ \\
\hline High N/C ratio & + & + & + & + & & & + & + & + & + & + & + & + & + & + & & & $13(77 \%)$ \\
\hline Necrosis & & & & + & + & + & + & + & & & & & + & + & + & & & $8(47 \%)$ \\
\hline 2-cell population & & & & + & & & & + & & & & + & + & + & + & + & + & $8(47 \%)$ \\
\hline Hypercellularity & & & + & + & & & & + & + & + & & + & & & + & & & $7(41 \%)$ \\
\hline $\begin{array}{l}\text { Clear/microvesicular } \\
\text { cytoplasm }\end{array}$ & & & + & & + & + & & + & + & + & & & & & & & & $6(35 \%)$ \\
\hline Cytoplasmic bile & & & & + & & & + & & & & & & & + & + & + & + & $6(35 \%)$ \\
\hline Nuclear irregularity & & & + & & + & + & & & + & + & & + & & & & & & $6(35 \%)$ \\
\hline Anisonucleosis & & & & + & + & & & + & & & & + & & & & + & + & $6(35 \%)$ \\
\hline Papillary groups & & & & + & + & + & + & & & + & & & & & & & & $5(29 \%)$ \\
\hline Multinucleated tumor cells & & & & + & & + & + & & + & & & & & & & & & $4(24 \%)$ \\
\hline Endothelial wrapping & & & & + & & & & + & & & & + & & & + & & & $4(24 \%)$ \\
\hline Ancillary studies (IHC/reticulin) & & & & + & + & + & + & + & + & + & + & & + & + & & & & $10(59 \%)$ \\
\hline
\end{tabular}

Abbreviations: IHC, immunohistochemistry (immunohistochemical stains including hepatocyte paraffin (Hep-Par), glypican 3, and arginase); N/C ratio, nuclear/ cytoplasmic ratio.

${ }^{a}$ Samples 5 and 6 were from the same patient, samples 9 and 10 were from the same patient, and samples 16 and 17 were from the same patient.

be positive on FISH were poorly differentiated, 1 tumor measured $8.0 \mathrm{~cm}$ and the other measured $11.3 \mathrm{~cm}$, and both patients had chronic viral hepatitis.

\section{Follow-Up Information}

Follow-up information was available for all patients and ranged from 0.2 months to 49 months, with a median of 3.5 months. Ten patients (71\%) died of disease, 3 patients $(21 \%)$ were alive with disease at the time of last follow-up, and 1 patient (7\%) was lost to follow-up at 49 months. It is interesting to note that of the 10 patients who died, the median survival was 3.5 months (range, $1-46$ months) and 6 of these patients (60\%) died 1 to 5 months after diagnosis. Of the 6 patients who died within 5 months of diagnosis, 1 patient had a very small $(2.0 \mathrm{~cm})$ and purely intraductal HCC.

\section{DISCUSSION}

To the best of our knowledge, the extension of HCC into the intrahepatic and extrahepatic biliary tree first was described in 1947 by Mallory et $\mathrm{al}^{15}$ and is known historically by names such as "icteric-type hepatoma" 16 and "cholestatic HCC." 17 When this occurs, it leads to the patient presenting with jaundice, elevated serum bilirubin, and elevated liver enzymes, symptoms and signs that were observed in greater than one-half of the patients in the current study cohort. Serum $\alpha$-fetoprotein may or may not be elevated, which, in the absence of a liver mass, may delay diagnosis and confound radiologists and gastroenterologists. Obstructive jaundice as the main presentation in patients with HCC occurs in $1 \%$ to $12 \%$ of patients. ${ }^{18}$ Early identification is important because patients may benefit from early surgical intervention. ${ }^{19}$

Ductal obstruction in HCC is due either to the intraductal accumulation of clots (hemobilia) or debris or intraductal tumor growth, duct wall invasion, or external compression by an expansile tumor. ${ }^{19}$ The intraductal component may be contiguous with the hepatic tumor or may be completely separate, with or without mucosal attachment. ${ }^{20}$ There even are reports of HCC occurring entirely within the biliary tree without a discernable hepatic component, independent of size and differentiation. ${ }^{21-23}$ Several of the cases in the current study were morphologically well differentiated, nearly one-half of the tumors measured $\leq 5.0 \mathrm{~cm}$, and $14 \%$ demonstrated pure intraductal growth. To our knowledge, the majority of intraductal tumors are pure HCCs, but mixed HCC-cholangiocarcinomas also have been described. ${ }^{24,25}$ Ductal involvement by HCC is not easily identifiable on computed tomography and magnetic resonance imaging but is more obvious on ERCP, which also allows for direct visualization and sampling of polypoid masses or strictures. ${ }^{19}$ Because of its rarity and subtle appearance, tumors either are missed by gastroenterologists or misinterpreted as cholangiocarcinoma 
or choledocholithiasis. ${ }^{26,27}$ Intraductal involvement by HCC is associated with a poor prognosis, ${ }^{19}$ which was observed in the current study, in which $70 \%$ of the study cohort died within 6 months of diagnosis.

The presence of HCC in BDBs also has been described in isolated reports, with the current study representing what to our knowledge is the largest series to date in the cytology literature. ${ }^{9-11,25,28}$ The most frequent cytologic features that we identified herein were singly dispersed, intact, and 3-dimensional clusters of polygonal tumor cells with well-defined cell borders; a relatively high nuclear/cytoplasmic ratio; abundant granular oncocytoid cytoplasm; and round to oval central nuclei with variable chromatin (hyperchromatic and hypochromatic) and prominent, often macro-, nucleoli. Widened trabeculae (which we defined as hepatic plates that were $>2$ cells in thickness) with clearly delineated endothelial lining cells were observed only rarely, as was intracytoplasmic bile, features that typically would favor HCC over cholangiocarcinoma. The fact that the majority of the samples in the current study were liquid-based preparations and had limited cellularity as well as necrosis may have contributed to the difficulty in finding intact trabeculae and clinging endothelial cells. These features were best seen on cell blocks but the cell blocks often were paucicellular and bloody. In addition, because of their monotony, more well-differentiated examples of HCC may be mistakenly classified as benign or indeterminate on BDBs, which happened in 3 of the cases in the current study. Although the majority of cases in the current study were diagnosed accurately on cytology, approximately $60 \%$ required ancillary immunocytochemical stains, including arginase and Hep-Par to confirm their hepatocellular phenotype and/or reticulin (Fig. 4) to confirm hepatic plate expansion.

HCC in BDBs must be distinguished from other lesions that more typically involve the intrahepatic and extrahepatic biliary tree and potentially could mimic HCC morphologically. By virtue of its intraductal location, HCC must be distinguished from an invasive cholangiocarcinoma, which is far more frequent at this anatomic site. Although the intracytoplasmic mucin vacuoles that are typical of adenocarcinoma were not noted in any of the cases in the current study, cytoplasmic clearing (clear cell or steatohepatitic features) was present in some samples, and potentially could cause confusion with cholangiocarcinoma, as it did in one case herein. Tumor cell negativity for hepatocellular immunocytochemical markers as well as the absence of cytoplasmic bile pigment should help with distinguishing between the two entities. However, mixed HCC-cholangiocarcinoma may be impossible to distinguish from pure $\mathrm{HCC}$ in $\mathrm{BDBs}$ and may require examination of a larger sample. Other intraductal neoplasms that may demonstrate eosinophilic cells similar to those of HCC include intraductal papillary neoplasm of the bile duct, particularly the oncocytic type, and oncocytic-type intraductal papillary mucinous neoplasm of the pancreas, which may involve the biliary tree. ${ }^{29-32}$ Both are characterized by papillary units lined by oncocytic cells with large nuclei, prominent nucleoli, and little if any cytoplasmic mucin. These features may mimic the papillae observed in some of the current study cases. Endothelial wrapping, cytoplasmic bile pigment, and positive hepatocellular markers would favor HCC. Well-differentiated neuroendocrine tumors often metastasize to the liver or may involve bile ducts, and when oncocytic they may mimic HCC. ${ }^{33,34}$ Unlike HCC, well-differentiated neuroendocrine tumors have eccentric nuclei and salt-and-pepper chromatin, and stain with neuroendocrine markers. ${ }^{34}$ The distinction between well-differentiated HCC and normal hepatocytes, which also may be sampled on BDBs, can be especially challenging. Identifying widened trabeculae with endothelial wrapping, nuclear pleomorphism, and macronucleoli should favor HCC over benign hepatic parenchyma, but this distinction ultimately may require biopsy. ${ }^{13,14}$

When HCC involves the biliary tree, it can lead to obstructive jaundice, ductal dilatation, and liver enzyme abnormalities, requiring ERCP and BDB, which may inadvertently sample these tumors. BDBs are characterized by cells with naked nuclei or abundant granular cytoplasm, a high nuclear/cytoplasmic ratio, large nuclei, and macronucleoli. The classic cytologic features of HCC usually noted on aspiration (endothelial wrapping, widened trabeculae, and cytoplasmic bile) are infrequent findings when using this sampling modality. To avoid misdiagnosis, cytopathologists should have a high index of suspicion when evaluating these samples, particularly in patients with concomitant liver lesions or known risk factors for HCC.

\section{FUNDING SUPPORT}

No specific funding was disclosed. 


\section{CONFLICT OF INTEREST DISCLOSURES}

The authors made no disclosures.

\section{AUTHOR CONTRIBUTIONS}

Shristi Bhattarai: Data curation, formal analysis, methodology, and writing-review and editing. Rondell P. Graham: Case contribution, data curation, formal analysis, investigation, methodology, and writing-review and editing. Carlie S. Sigel: Case contribution, data curation, formal analysis, investigation, methodology, and writing-review and editing. Jiaqi Shi: Case contribution, data curation, formal analysis, investigation, methodology, and writing-review and editing. Raul S. Gonzalez: Case contribution, data curation, formal analysis, investigation, methodology, and writing-review and editing. Yue Xue: Case contribution, formal analysis, investigation, methodology, and writing-review and editing. Alyssa M. Krasinskas: Case contribution and writing-review and editing. Kim HooKim: Formal analysis, investigation, methodology, and writing-review and editing. Volkan Adsay: Case contribution, formal analysis, methodology, and writing-review and editing. Michelle D. Reid: Case contribution, conceptualization, data curation, formal analysis, writing-original draft, writingreview and editing, and supervision.

\section{REFERENCES}

1. Layfield LJ, Wax TD, Lee JG, Cotton PB. Accuracy and morphologic aspects of pancreatic and biliary duct brushings. Acta Cytol. 1995;39:11-18.

2. Logrono R, Kurtycz D, Molina C, Trivedi V, Wong J, Block K. Analysis of false-negative diagnoses on endoscopic brush cytology of biliary and pancreatic duct strictures. Arch Pathol Lab Med. 2000;124:387-392.

3. Choi WT, Swanson PE, Grieco VS, Wang D, Westerhoff M. The outcomes of "atypical" and "suspicious" bile duct brushings in the identification of pancreaticobiliary tumors: follow-up analysis of surgical resection specimens. Diagn Cytopathol. 2015;43:885-891.

4. Okonkwo AM, De Frias DV, Gunn R, et al. Reclassification of "atypical" diagnoses in endoscopic retrograde cholangiopancreaticographyguided biliary brushings. Acta Cytol. 2003;47:435-442.

5. Volmar KE, Vollmer RT, Routbort MJ, Creager AJ. Pancreatic and bile duct brushing cytology in 1000 cases: review of findings and comparison of preparation methods. Cancer. 2006;108:231-238.

6. Hacihasanoglu E, Memis B, Pehlivanoglu B, et al. Factors impacting the performance characteristics of bile duct brushings: a clinico-cytopathologic analysis of 253 patients. Arch Pathol Lab Med. 2018;142:863-870.

7. Rupp M, Hawthorne CM, Ehya H. Brushing cytology in biliary tract obstruction. Acta Cytol. 1990;34:221-226.

8. Vadmal MS, Byrne-Semmelmeier S, Smilari TF, Hajdu SI. Biliary tract brush cytology. Acta Cytol. 2000;44:533-538.

9. Dusenbery D. Biliary stricture due to hepatocellular carcinoma: diagnosis by bile duct brushing cytology. Diagn Cytopathol. 1997;16:55-56.

10. Schmelzle M, Matthaei H, Lehwald N, et al. Extrahepatic intraductal ectopic hepatocellular carcinoma: bile duct filling defect. Hepatobiliary Pancreat Dis Int. 2009;8:650-652.

11. Fu LY, Mitchell KA, Cai G. Clear cell hepatocellular carcinoma diagnosed by bile duct brushing cytology. Diagn Cytopathol. 2016;44:147-151.

12. Torbenson MS, Ng IOL, Park YN, Roncalli M, Sakamato M. Hepatocellular carcinoma. In: WHO Classification of Tumours Editorial Board, ed. WHO Classification of Tumours: Digestive System Tumours. 5th ed. IARC Press; 2019:229-239.
13. Das DK. Cytodiagnosis of hepatocellular carcinoma in fine-needle aspirates of the liver: its differentiation from reactive hepatocytes and metastatic adenocarcinoma. Diagn Cytopathol. 1999;21:370-377.

14. de Boer WB, Segal A, Frost FA, Sterrett GF. Cytodiagnosis of well differentiated hepatocellular carcinoma: can indeterminate diagnoses be reduced? Cancer. 1999;87:270-277.

15. Mallory TB, Castleman B, Parris BE. Case records of the Massachusetts General Hospital. N Engl J Med. 1947;237:673-676.

16. Lin TY, Chen KM, Chen YR, Lin WS, Wang TH, Sung JL. Icteric type hepatoma. Med Chir Dig. 1975;4:267-270.

17. Okuda K. Clinical aspects of hepatocellular carcinoma: analysis of 134 cases. In: Okuda K, Peters RL, eds. Hepatocellular Carcinoma. John Wiley \& Sons; 1976:387-436.

18. Kew MC, Paterson AC. Unusual clinical presentations of hepatocellular carcinoma. Trop Gastroenterol. 1985;6:10-22.

19. Qin LX, Tang ZY. Hepatocellular carcinoma with obstructive jaundice: diagnosis, treatment and prognosis. World J Gastroenterol. 2003;9:385-391.

20. Narita R, Oto T, Mimura Y, et al. Biliary obstruction caused by intrabiliary transplantation from hepatocellular carcinoma. J Gastroenterol. 2002;37:55-58.

21. Buckmaster MJ, Schwartz RW, Carnahan GE, Strodel WE. Hepatocellular carcinoma embolus to the common hepatic duct with no detectable primary hepatic tumor. Am Surg. 1994;60:699-702.

22. Cho HG, Chung JP, Lee KS, et al. Extrahepatic bile duct hepatocellular carcinoma without primary hepatic parenchymal lesions-a case report. Korean J Intern Med. 1996;11:169-174.

23. Soyer P, Laissy JP, Bluemke DA, Sibert A, Menu Y. Bile duct involvement in hepatocellular carcinoma: MR demonstration. Abdom Imaging. 1995;20:118-121.

24. Saito M, Hige S, Takeda H, Tomaru U, Shibata M, Asaka M. Combined hepatocellular carcinoma and cholangiocarcinoma growing into the common bile duct. J Gastroenterol. 2001;36:842-847.

25. Dusenbery D. Combined hepatocellular-cholangiocarcinoma. Cytologic findings in four cases. Acta Cytol. 1997;41:903-909.

26. Jan YY, Chen MF. Obstructive jaundice secondary to hepatocellular carcinoma rupture into the common bile duct: choledochoscopic findings. Hepatogastroenterology. 1999;46:157-161.

27. Kojiro M, Kawabata K, Kawano Y, Shirai F, Takemoto N, Nakashima T. Hepatocellular carcinoma presenting as intrabile duct tumor growth: a clinicopathologic study of 24 cases. Cancer. 1982;49: 2144-2147.

28. Kunz G Jr, Chung J, Ali SZ. Hepatocellular carcinoma-fibrolamellar variant: cytopathology of an unusual case. Diagn Cytopathol. 2002;26:257-261.

29. Reddy K, Hooper K, Frost A, et al. Intraductal papillary carcinoma of common bile duct diagnosed by endoscopic ultrasound-guided fine-needle aspiration. Endoscopy. 2014;46(suppl 1 UCTN):E248-E249.

30. Reid MD, Stallworth CR, Lewis MM, et al. Cytopathologic diagnosis of oncocytic type intraductal papillary mucinous neoplasm: criteria and clinical implications of accurate diagnosis. Cancer Cytopathol. 2016;124:122-134.

31. Jurczyk MF, Zhu B, Villa C, DeFrias D, Lin X. Cytomorphology of intraductal oncocytic papillary neoplasm of the liver. Diagn Cytopathol. 2014;42:895-898.

32. Bansal A, Thung SN, Zhu H, Schwartz M, Lewis S. Synchronous pancreatic adenocarcinoma and intrahepatic cholangiocarcinoma arising in the context of intraductal papillary neoplasms. Clin Imaging. 2016;40:897-901.

33. Volante M, La Rosa S, Castellano I, Finzi G, Capella C, Bussolati G. Clinico-pathological features of a series of 11 oncocytic endocrine tumours of the pancreas. Virchows Arch. 2006;448:545-551.

34. Chen S, Wang X, Lin J. Fine needle aspiration of oncocytic variants of pancreatic neuroendocrine tumor: a report of three misdiagnosed cases. Acta Cytol. 2014;58:131-137. 